Article

\title{
Supplemental Aspergillus Lipase and Protease Preparations Display Powerful Bifidogenic Effects and Modulate the Gut Microbiota Community of Rats
}

\author{
Yongshou Yang ${ }^{1,2}$, Thanutchaporn Kumrungsee ${ }^{2, * \mathbb{C}}$, Norihisa Kato ${ }^{2, *} \mathbb{C}$, Shinji Fukuda ${ }^{3,4,5} \mathbb{D}^{\text {, Manabu Kuroda }}{ }^{6}$ \\ and Shotaro Yamaguchi ${ }^{6}$ \\ 1 School of Life Sciences, Anhui University, Hefei 230036, China; yangyongshou@g.ecc.u-tokyo.ac.jp \\ Graduate School of Integrated Sciences for Life, Hiroshima University, Higashi-Hiroshima 739-8528, Japan \\ Institute for Advanced Biosciences, Keio University, Tsuruoka 997-0052, Japan; sfukuda@sfc.keio.ac.jp \\ Transborder Medical Research Center, Faculty of Medicine, University of Tsukuba, Tsukuba 305-8575, Japan \\ Gut Environmental Design Group, Kanagawa Institute of Industrial Science and Technology, \\ Kawasaki 210-0821, Japan \\ 6 Amano Enzyme Inc., Nagoya 460-8630, Japan; manabu_kuroda@amano-enzyme.com (M.K.); \\ shotaro_yamaguchi@amano-enzyme.com (S.Y.) \\ * Correspondence: kumrung@hiroshima-u.ac.jp (T.K.); nkato@hiroshima-u.ac.jp (N.K.); \\ Tel.: +81-82-424-7980 (T.K.); +81-82-426-0774 (N.K.)
}

check for updates

Citation: Yang, Y.; Kumrungsee, T.; Kato, N.; Fukuda, S.; Kuroda, M.; Yamaguchi, S. Supplemental Aspergillus Lipase and Protease Preparations Display Powerful Bifidogenic Effects and Modulate the Gut Microbiota Community of Rats. Fermentation 2021, 7, 294. https:// doi.org/10.3390/fermentation7040294

Academic Editor: Hiroshi Kitagaki

Received: 4 November 2021

Accepted: 29 November 2021

Published: 1 December 2021

Publisher's Note: MDPI stays neutral with regard to jurisdictional claims in published maps and institutional affiliations.

Copyright: (c) 2021 by the authors. Licensee MDPI, Basel, Switzerland. This article is an open access article distributed under the terms and conditions of the Creative Commons Attribution (CC BY) license (https:/ / creativecommons.org/licenses/by/ $4.0 /)$.
Abstract: Aspergillus-derived protease and lipase, which are involved in the production of Aspergillusfermented foods, are consumed as digestive enzyme supplements. A marked bifidogenic effect of supplemental Aspergillus protease preparation (AP) in rats fed with a high-fat diet was identified. This study was conducted to examine whether the consumption of Aspergillus-derived lipase exerts similar bifidogenic effect. Rats were fed diets supplemented with either an Aspergillus-derived lipase preparation (AL) or AP at $0.1 \%$ for two weeks. $16 \mathrm{~S}$ rRNA gene sequencing analysis indicated that supplemental AL and AP markedly influenced cecal microbial community. At the phylum level, treatment with AL and AP resulted in a lower relative abundance of Firmicutes and Bacteroidetes, but a higher relative abundance of Actinobacteria and Proteobacteria than the control rats $(p<0.05)$. At the genus level, AL and AP remarkedly elevated the relative abundances of Bifidobacterium, Collinsella, and Enterococcus, but significantly reduced those of Oscillospira, Dorea, and Coprobacillus $(p<0.05)$. These modulations were similar to those reported by several studies with typical prebiotic oligosaccharides. Notably, the bifidogenic effect of AL was much greater than that of AP. Our results show that the two different Aspergillus-derived preparations, AL and AP, have strong bifidogenic effects and can change the microbiota's composition.

Keywords: Aspergillus; lipase; protease; bifidogenic effect; prebiotics; 16S rRNA gene sequencing

\section{Introduction}

The gut microbiota has recently received increasing attention due to its important roles in host metabolism, physiology, and immune system development. Among many environmental factors, diet is considered a key factor influencing the composition and function of the gut microbiota [1]. For instance, a western diet, characterized by high-fat and low dietary fiber, affects the gut microbiota's composition and causes gut bacterial dysbiosis. Furthermore, an altered gut microbiota is associated with system inflammation and higher incidence of chronic diseases, such as inflammatory bowel disease (IBD), cardiovascular disease, and diabetes [2]. However, increasing evidence indicates that dietary intervention and particular prebiotics supplementation, can reverse high-fat-induced gut dysbiosis. Short-chain nondigestible carbohydrates, such as fructooligosaccharides (FOS, an inulin-type fructan), and galactooligosaccharides (GOS) are well-known prebiotics that 
can increase populations of certain beneficial bacteria, typically Bifidobacterium and Lactobacillus species [3]. By modulating imbalances in the gut microbiota, prebiotics have been shown to promote gut health and decrease the risk of bowel and systemic diseases $[4,5]$.

The fungi genus Aspergillus contains many species found in various ecological niches. Some nonpathogenic species, such as Aspergillus oryzae and A. niger, have a long history of application in food fermentation industries in Japan. During Aspergillus-associated fermentation, extracellular hydrolytic enzymes, such as proteases, $\alpha$-amylases, and lipases, are released into the reaction system. They are responsible for the fermentation and release of umami and flavor substances [6,7]. Extracted Aspergillus enzymes, such as proteases and lipases, have also been used in food processing [7]. Commercially available digestive supplements containing proteases, $\alpha$-amylases, and lipases derived from microbial species have been manufactured to treat insufficient food digestion [8]. A previous study of ours found that dietary supplementation with $A$. oryzae-derived protease preparation (AP) and the purified acid protease markedly elevated cecal Bifidobacterium spp. levels in rats fed a high-fat (HF) diet [9-11]. Cecal levels of organic acids and fecal levels of immunoglobulin $\mathrm{A}(\mathrm{IgA})$ and mucins were also increased by AP consumption, whereas $\mathrm{pH}$ in the cecal contents was significantly decreased [9]. The increased abundance of Bifidobacterium spp. in the presence of Aspergillus protease has been proposed to be mediated by the increased availability of free amino acids following hydrolysis of undigested proteins in the large intestine [11]. Recently, preliminary investigations into the bifidogenic effects of several digestive enzyme preparations for lipids and carbohydrates derived from Aspergillus spp. have been completed. Results of the quantitative polymerase chain reaction (qPCR) analysis indicated a striking increase in cecal abundance of Bifidobacterium spp. in rats following treatment with Aspergillus-derived lipase preparation (AL). To our knowledge, there is limited information on how dietary exogenous lipase supplementation modulates the gut environment. It was hypothesized that dietary supplemental AL would exert bifidogenic effects in a manner similar to that of the AP. Thus, this study examines the effect of AL on the gut microbiota and compares those with the effects of AP in rats fed a HF diet. The study was conducted using rats fed with HF diet since HF diet-induced colon dysbiosis, inflammation, and diseases have been reported to be suppressed by dietary prebiotic oligosaccharides [12].

\section{Materials and Methods}

\subsection{Animals and Diets}

Twenty-three male Sprague Dawley rats (four weeks old) were purchased from Charles River Japan. The rats were individually housed in cages in a controlled temperature environment $\left(23 \pm 2{ }^{\circ} \mathrm{C}\right), 12 \mathrm{~h}$ light-dark cycle, and relative humidity of $50-60 \%$. The experimental animals were acclimatized for 7 days and then randomly divided into the following three groups based on their diet: HF diet (Ctrl: Control, Table S1; eight rats) [9]; HF diet $+0.1 \%(w / w) A$. niger-derived lipase preparation (AL: Lipase AP12, lipase activity at $\mathrm{pH}$ 6.0, 60,000 U/g; Amano Enzyme Inc. Nagoya, Japan; eight rats), and HF diet + $0.1 \%(w / w)$ A. oryzae-derived protease preparation (AP: Protease A "Amano" SD, protease activity at pH 6.0, 100,000 U/g; Amano Enzyme Inc. Nagoya, Japan; seven rats). For two weeks, equal amounts of the experimental diets were given daily in food cups at $18: 00 \mathrm{~h}(9,10,12,14$, and $15 \mathrm{~g}$ on days $1,2-4,5-7,8-12$, and $13-14$, respectively) to prevent differences in food intake. All diets were consumed daily. The rats had ad libitum access to fresh water. The study protocols (protocol identical No. C15-12) were approved by the Ethics Committee of the Hiroshima University.

\subsection{Sample Collection}

At the end of the two-week treatment period, the rats were anesthetized (13:00-15:00 h) by inhalation of isoflurane in a desiccator to minimize suffering, and then euthanized by decapitation. The cecum was immediately excised, and its contents were completely 
removed weighed, and stored at $-80^{\circ} \mathrm{C}$ until subsequent analysis of cecal microbiota and organic acids.

\subsection{S rRNA Gene-Based Microbiome Analysis}

Total bacterial DNA in cecal contents was extracted using the QIAamp Stool Mini Kit according to the manufacturer's instructions (QIAGEN, Hilden, Germany). Then, extracted bacterial DNA was quantified using NanoDrop spectrometry (NanoDrop Technology, Wilmington, DL, USA). The V1-V2 region of the $16 \mathrm{~S}$ rRNA genes was amplified from the DNA isolated from cecal contents using the following bacterial universal primer set: $27 \mathrm{~F}$ (5'-ACACTCTTTCCCTACACGACGCTCTTCCGATCTAGRGTTTGATYMTGGCTCAG-3') and 338R(5'-GTGACTGGAGTTCAGACGTGTGCTCTTCCGATCTTGCTGCCTCCCGTAG GAGT-3'). The following library preparation was performed as described previously [13]. Finally, all the barcoded V1-V2 PCR amplicons were sequenced using Illumina MiSeq sequencing technology at a read length of $2 \times 300 \mathrm{bp}$ based on the manufacturer's instructions (Illumina, San Diego, CA, USA).

\subsection{Bioinformatics Analysis}

First, fast-length adjustment of short reads (v.1.2.11) [14] was used to assemble the paired-end reads. Assembled reads with an average $Q$-value $<25$ were filtered out using an in-house script. The same numbers of filtered reads were randomly selected from each sample and used for further analysis. The selected reads were then processed using QIIME pipeline (ver. 1.9.1). The high-quality sequences were clustered into operational taxonomic units (OTUs) at 97\% sequence similarity, and OTUs were assigned to the Greengenes database.

\subsection{Analysis of Cecal Organic Acids}

The concentrations of organic acids in cecal contents were measured by gas chromatography/mass spectrometry as previously described [15].

\subsection{Data Analysis}

Data are expressed as mean \pm standard error or boxplots with minimum, maximum and median. Statistical analysis was performed using one-way analysis of variance (ANOVA) and Tukey-Kramer HSD test (Figures 1 and 3-5). The data separation in the principal coordinate analysis ordination of beta-diversity was conducted by the PERMANOVA permutation-based statistical test in vegan package of $\mathrm{R}$, and $p$-values were generated based on 999 permutations (Figure 1). Some bacterial taxa data were subjected to linear discriminant analysis effect size (LEfSe) analysis, which uses the two-tailed nonparametric Kruskal-Wallis test to evaluate the significance of differences between taxa (Figure 2). $p<0.05$ was considered to indicate a statistically significant different. For the relationship between organic acids and microbiota composition, Pearson's correlation coefficient (r) was calculated, and the resulting correlation matrix was visualized by using $\mathrm{R}$ software (version 4.0.2) (Figure S1).

\subsection{Evaluation of the Risk of Bias in the Methodology}

The risk of bias of this study was assessed using the Systematic Review Centre for Laboratory Animal Experimentation (SYRCLE) risk of bias (RoB) tool [16]. Two independent authors (YY and NK) evaluated the following nine items: (1) sequence generation, (2) baseline characteristics, (3) allocation concealment, (4) random housing, (5) intervention blinding, (6) random outcome assessment, (7) outcome blinding, (8) incomplete outcome data, and (9) selective outcome reporting. All items were judged as 'yes' (low risk of bias) by two authors (YY and NK) independently. 


\section{Results}

\subsection{Food intake, Body Weight, and Cecal Content Weight}

Total food intake for the two-week experimental period and final body weight were unaffected by dietary treatment (data not shown, $p>0.05$ ). The AP and AL groups significantly increased in the weight of cecal contents compared to the Ctrl group $(4.72 \pm 0.39 \mathrm{~g}$, $3.53 \pm 0.36 \mathrm{~g}$, and $1.44 \pm 0.06 \mathrm{~g}$, respectively, $p<0.05$, Tukey-Kramer HSD test).

\subsection{Cecal Microbiota}

For microbiota analysis by $16 \mathrm{~S}$ rRNA gene sequencing, a total of 790,645 high-quality reads were passed using the QIIME filter. Unweighted and weighted UniFrac PCoA and PERMANOVA analyses were conducted to compare the microbial structure. The results of UniFrac PCoA and PERMANOVA analyses indicated that the microbial composition differed between the Ctrl and AL groups and between the Ctrl and AP groups (in both unweighted and weighted) (Figure $1 \mathrm{~A}, \mathrm{~B}, p<0.05$ ). However, the different alpha-diversity indices indicated a higher bacterial diversity in the Ctrl group than in the AL and AP groups (Figure 1C-E, $p<0.05$ ).

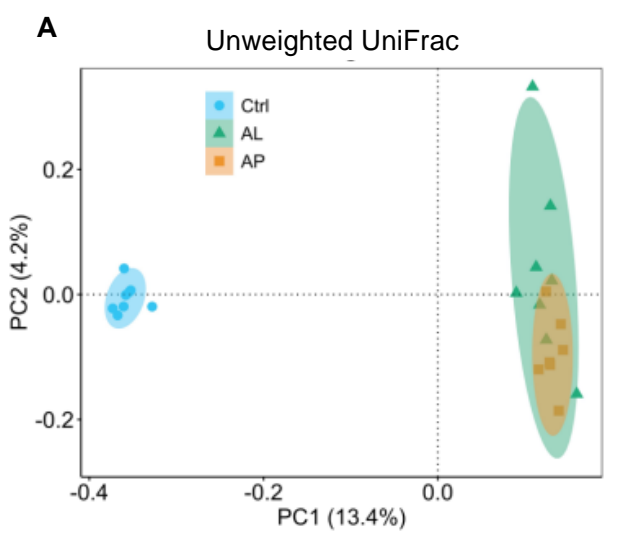

PERMANOVA

Ctrl vs AL: $p=0.002$

Ctrl vs AP: $p=0.002$

AL vs AP: $p=0.191$

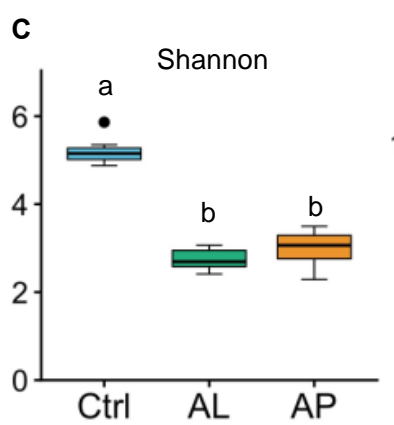

D

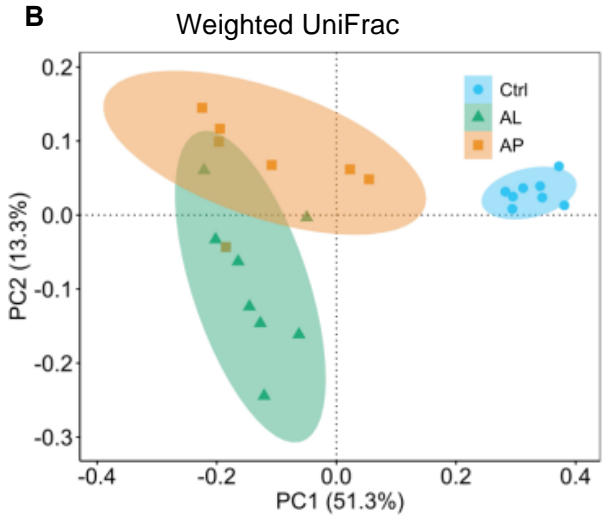

PERMANOVA

Ctrl vs AL: $p=0.002$

Ctrl vs AP: $p=0.002$

AL vs AP: $p=0.062$

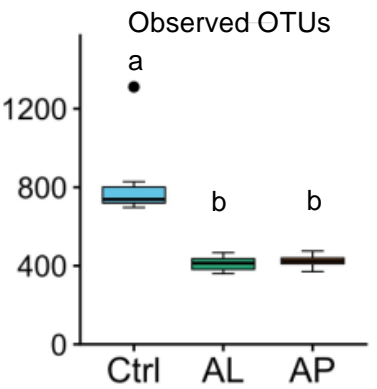

E

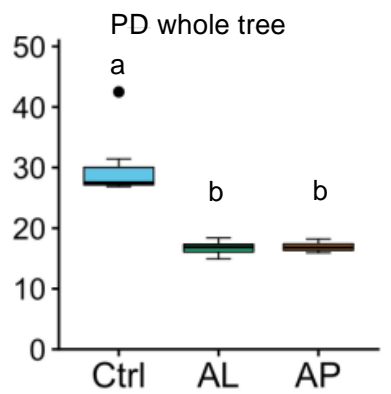

Figure 1. Effects of dietary treatment with $\mathrm{AL}$ and AP on cecal microbiome profiles and alphadiversity. 16S rRNA gene-based microbiome analysis was used to evaluate the effects. PCoA of unweighted (A) and weighted UniFrac (B) and PERMANOVA analysis were performed to compare the gut microbiome profiles of the experimental groups. The diversity of the gut microbiota within samples was measured by (C) Shannon index, (D) observed OTUs, and (E) PD whole tree. Superscript with different letters indicate significantly difference at $p<0.05$ (Tukey-Kramer HSD test).

LEfSe analysis results indicated that 45 bacterial taxa differed between the Ctrl and AL groups (Figure $2 \mathrm{~A}, p<0.05$ ), whereas 39 taxa varied between the $\mathrm{Ctrl}$ and AP groups 
(Figure 2B, $p<0.05$ ). This analysis identified the phylum Proteobacteria, Actinobacteria, Bacteroidetes, and Firmicute differed between the Ctrl and AL groups and between the Ctrl and AP groups. This analysis further identified bacterial species Collinsella, Lactobacillus, Bifidobacterium, Eggerthella, Enterococcus, Dehalobacterium, Coprobacillus, Dorea, Akkermansia, Adlercreutzia, rc4_4, Oscipillospira, Coprococcus, Allobaculum, Ruminococcus, Holdemania, Roseburia, and Parabacteroides varied between the Ctrl and AL groups and between the Ctrl and AP groups.
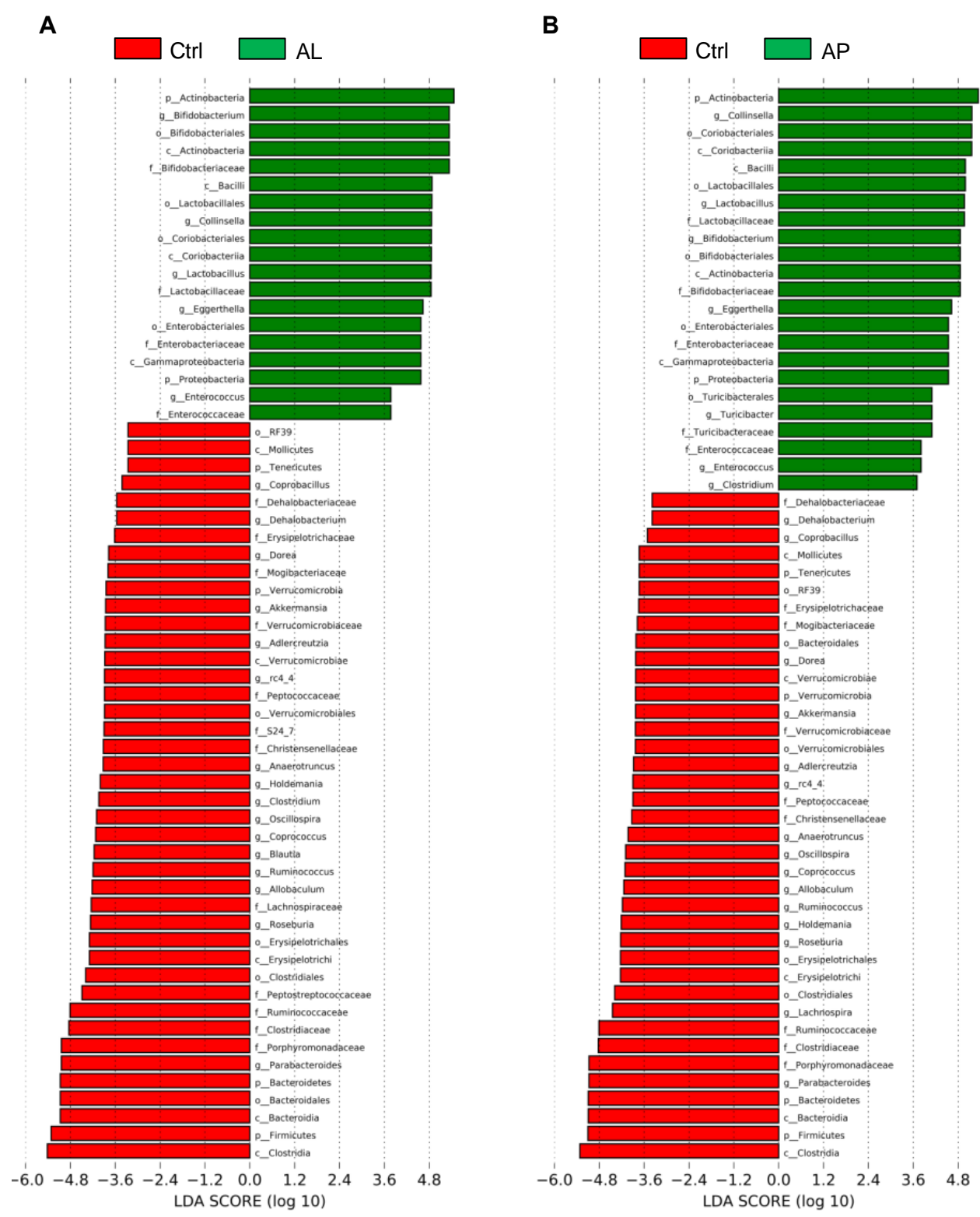

Figure 2. Comparison of the different taxa between the Ctrl and AL groups (A), and the Ctrl and AP groups (B) through LEfSe analysis. The two-tailed nonparametric Kruskal-Wallis test showed the significant differences in the taxa between the Ctrl group and the AL and AP groups $(p<0.05)$.

At the phylum level (Figure 3A), the relative abundances of Bacteroidetes and Firmicutes were significantly decreased by treatment with AL and AP, while the relative abundances of Proteobacteria and Actinobacteria were markedly increased, compared to Ctrl ( $p<0.05$, Tukey-Kramer HSD test). There was no difference in the phylum levels such as Bacteroides, Firmicute, Proteobaceria, and Actinobacteria between the AL and AP groups $(p>0.05)$. These changes were shown at the family level (Figure 3B), with 
significant decreases in the relative abundances of Clostridiaceae (phylum: Firmicute), Ruminococcaceae (phylum: Firmicute) and Porphyromonadaceae (phylum: Bacteroides), and significant increases in the relative abundances of Enterobacteriaceae (phylum: Proteobacteria), Coriobacteriaceae (phylum: Actinobacteria) and Bifidobacteriaceae (phylum: Actinobacteria) ( $p<0.05$, Tukey-Kramer HSD test). The levels of Lachnospiraceae (phylum: Firmicute) in the AL group were significantly lower than those in other groups $(p<0.05)$. The levels of Lactobacillaceae were unaffected $(p>0.05)$.
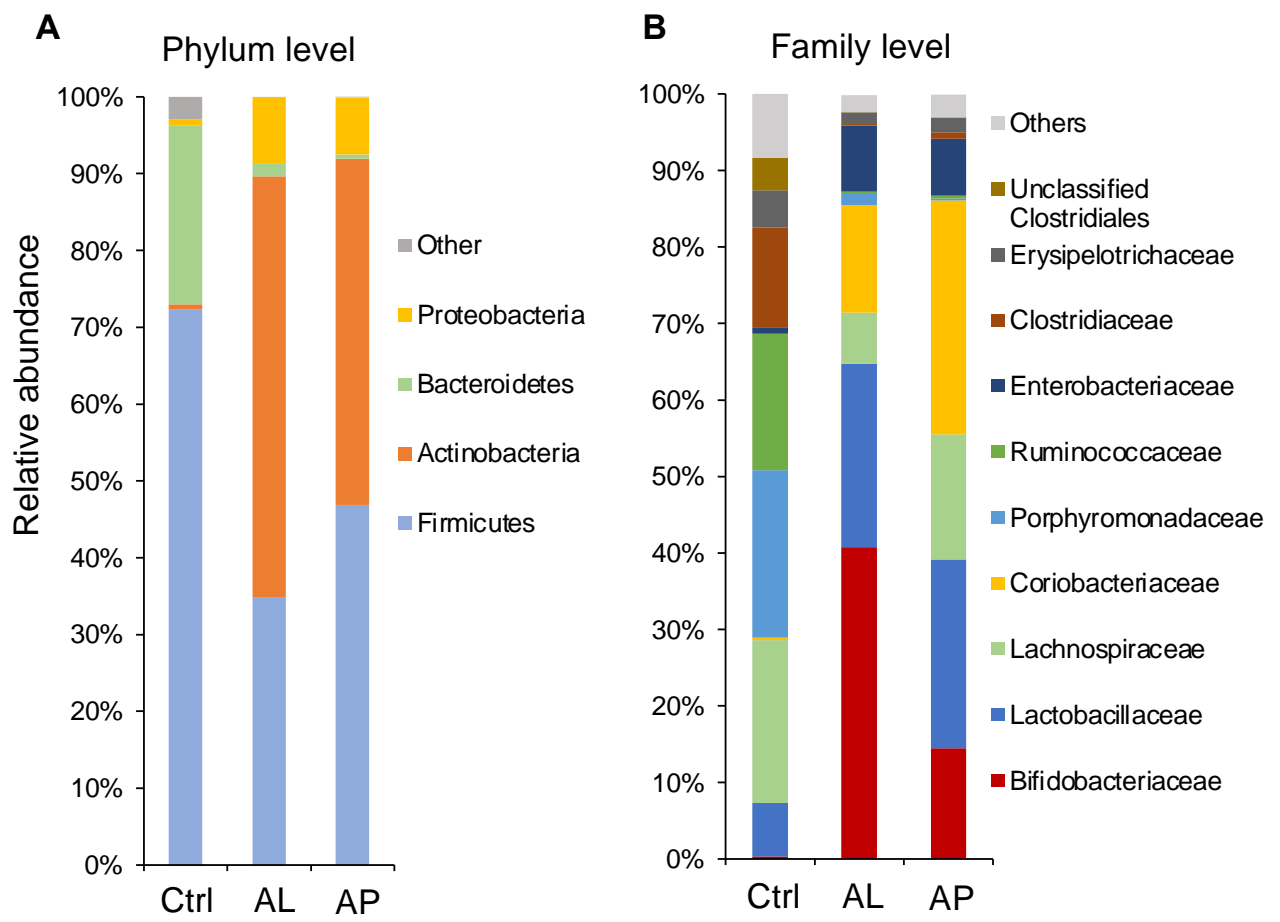

Figure 3. Effects of dietary AL and AP on cecal microbiota composition at the phylum (A) and family (B) levels.

At the genus level (Figure 4), AL and AP treatments strikingly increased the relative abundances of Bifidobacterium (family: Bifidobacteriaceae) by 120- and 43-fold on average, respectively, and Collinsella (family: Coriobacteriaceae) by 279 - and 609-fold on average, respectively $(p<0.05)$. The levels of Enterococcus (family: Enteroccaceae) were also significantly increased by dietary AL and AP (50- and 51-fold on average, respectively, $p<0.05$ ) (Figure 4). The relative abundances of Parabacteroides (family: Porphyromonadaceae), Allobaculum (family: Erysipelotrichaceae), Oscillospira (family: Ruminococcaceae), and Dorea (family: Lachnospiraceae) were significantly lower in the AL and AP groups $(p<0.05)$. Coprobacillus (family: Erysipelotrichaceae) was detected in all the Ctrl rats, but not detectable in the AL and AP rats. A significant increase in the Bifidobacterium counts by $\mathrm{AL}$ and AP was also observed when expressed as the copy numbers per $g$ wet weight of cecal contents and per total cecal contents (qPCR analysis, data not shown). The relative abundances of Lactobacillus species were not affected by dietary treatment $(p>0.05)$. The relative abundances of Bifidobacterium in the AL group were much higher than those in the AP group $(p<0.05)$, while those of Collinsella in the AL group were significantly lower than those in the AP group $(p<0.05)$ (Figure 4). Treatment with AL and AP had no significant effect on other genus levels such as Eggerthella, Mucispirillum, Staphylococcus, Lactococcus, Turicibacter, Clostridium, Blautia, Coprococcus, Roseburia, Ruminococcus, rc4-4, Anaerotruncus, and Eubacterium (data not shown). The genera with a mean relative abundance $<0.2 \%$ in all groups were not considered for statistical analysis. 

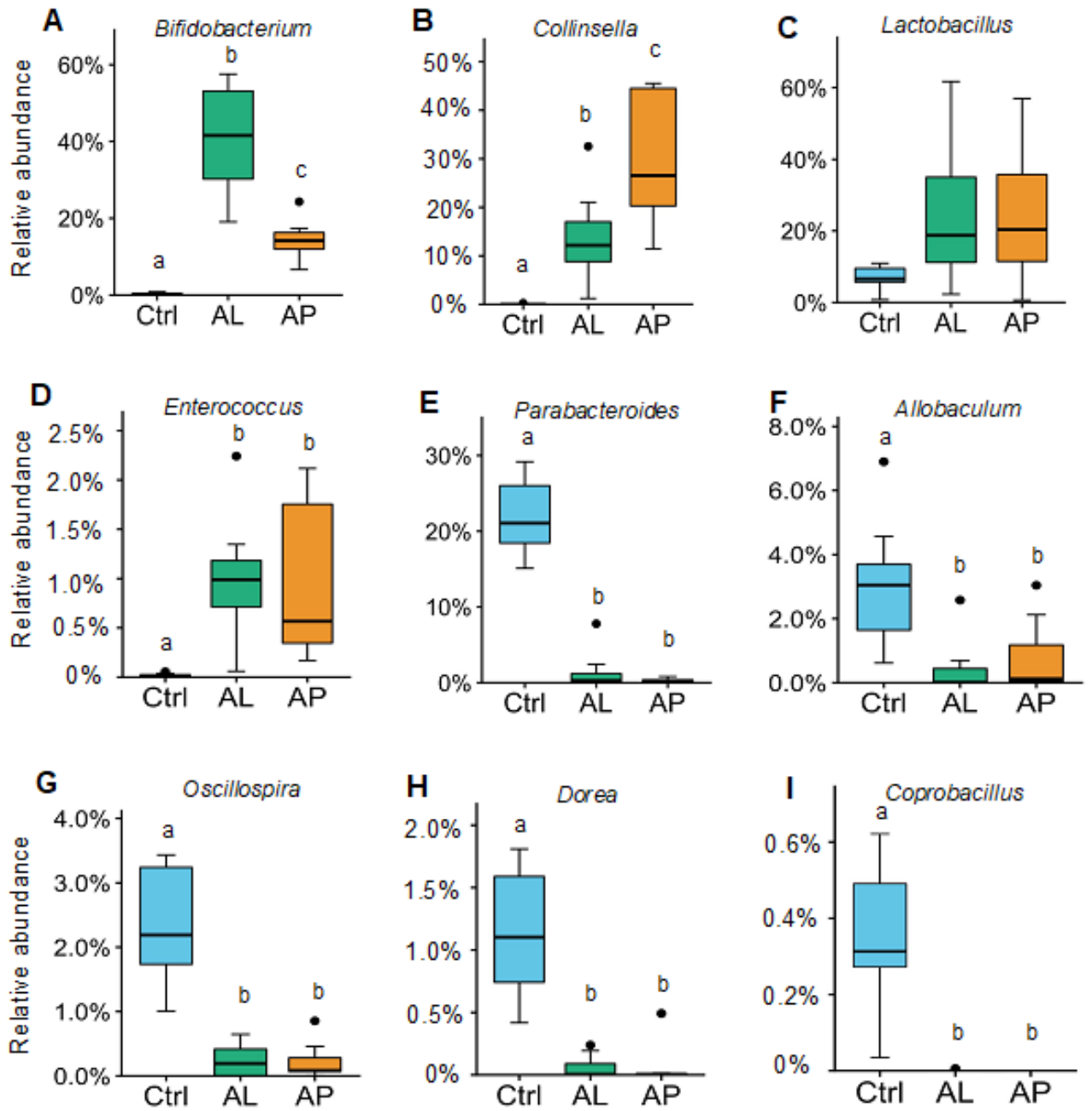

Figure 4. Effects of dietary $\mathrm{AL}$ and $\mathrm{AP}$ on cecal microbiota composition (A): Bifidobacterium, (B): Collinsella, (C): Lactobacillus, (D): Enteroccus, (E): Parabacteroides, (F): Allobaculum, (G): Oscillospira, (H): Dorea, and (I): Coprobacillus at the genus levels. Data are presented as a boxplot with median and min-max whiskers. The dots $(\bullet)$ in the boxplots are outliers. Superscript with different letters indicate significantly difference at $p<0.05$ (Tukey-Kramer HSD test).

\subsection{Cecal Organic Acids}

Figure 5 indicates the concentrations of cecal organic acids. Treatment with AL and AP markedly increased the concentrations of lactate (164- and 144-fold on average, respectively), but significantly decreased those of acetate (2.0- and 2.1-fold on average, respectively, propionate (50- and 25-fold on average, respectively), and butyrate (10- and 5.4-fold on average, respectively) $(p<0.05)$. Figure S1 further indicates the relationship between the levels of organic acids and various bacteria. The levels of lactate significantly correlated with the relative abundances of lactate-producing bacteria Bifidobacterium $(\mathrm{r}=0.688$, $p<0.001)$ and Enterococcus $(r=0.473, p<0.05)$. In general, the relative abundances of the genera such as Parabacteroides, Dorea, Oscillospira, Akkermansia, and Coprobacillus had a strong positive association with propionate levels, but a strong negative association with lactate levels (Figure S1). 
A

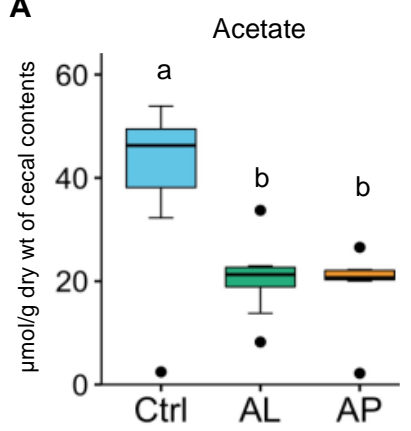

D

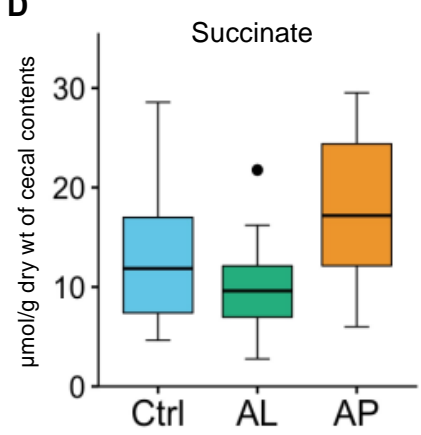

B

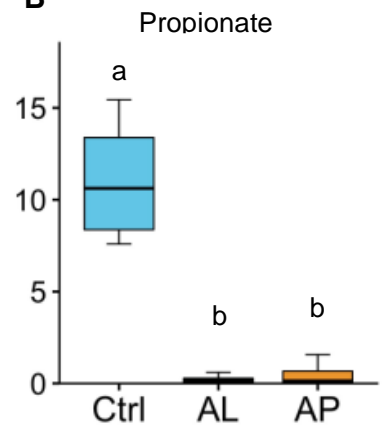

\section{E}

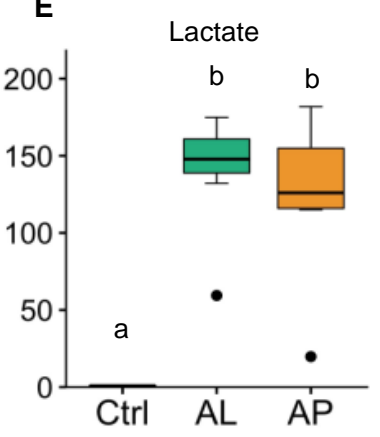

C
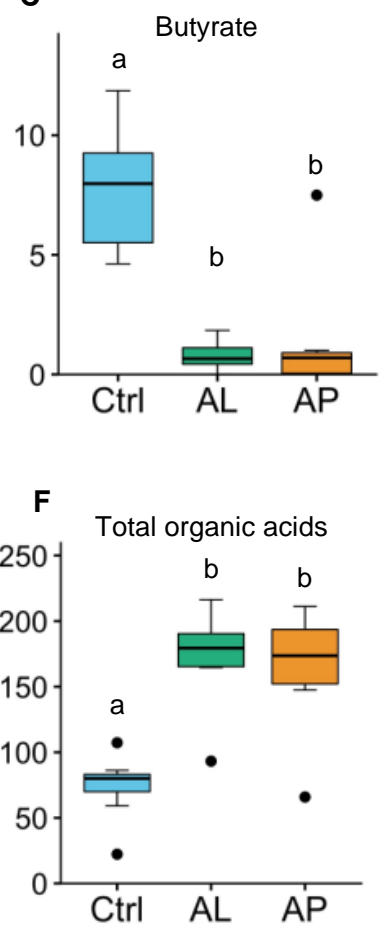

Figure 5. Effects of dietary AL and AP on cecal levels of organic acids (A): acetate, (B): propionate, (C): butyrate, (D): succinate, (E): lactate, and (F): total organic acids. Data are presented as a boxplot with median and min-max whiskers. The dots $(\bullet)$ in the boxplots are outliers. Superscript with different letters indicate significantly difference at $p<0.05$ (Tukey-Kramer HSD test).

\section{Discussion}

\subsection{Bifidobacterium}

This study provided the first evidence of the strong bifidogenic effects of AL in addition to AP. Intriguingly, the bifidogenic effect of AL was much stronger than that of AP. These results imply that the bifidogenic effect is not specific for Aspergillus protease, and also might be possible for other hydrolases, such as Aspergillus lipase. Aspergillus lipase and protease preparations used in this study are often included in commercially available digestive enzyme supplements to improve food digestion. Accordingly, the information on the effect of the enzyme preparations on gut health may be of practical relevance, and may also help in the understanding of the health benefits of Aspergillus-fermented foods containing Aspergillus lipases and proteases. Currently, several prebiotics are wellknown to confer health benefits through increasing probiotics [3,4]. To our knowledge, this study, together with previous studies [9-11], implies that the Aspergillus-derived lipase and protease may be considered as a new type of prebiotics, and introduce a new concept of "prebiotic digestive enzymes".

Our previous studies [10,11] found consumption of AP and purified acid protease obtained from A. oryzae markedly elevated cecal Bifidobacterium abundance. In contrast, the bifidogenic effect was absent in rats fed inactivated acid protease or inactivated AP, implying the involvement of protease activity in the mechanism of prebiotic activity. Additionally, treatment with AP increased cecal free amino acids, which correlated well with modulation of the relative abundance of Bifidobacterium [11]. Thus, we speculated that the increased levels of free amino acids (available amino acids) seen following consumption of AP promoted the growth of Bifidobacterium species. It is interesting to investigate whether an increased lipase activity in the gut arising from the supplemental AL could promote triglyceride hydrolysis to free long-chain fatty acids, which might increase the growth of Bifidobacterium species. The probiotic effects of Bifidobacterium are well-studied, imparting positive health benefits to the human host and preventing several disorders, 
such as gastrointestinal disorders, allergy, liver diseases, obesity, metabolic syndrome, cardiovascular diseases, cancers, autoimmune diseases, and brain diseases [3-5]. Due to their potential health-promoting properties, Bifidobacterium is incorporated into various foods as active ingredients. Many studies suggest that consumption of prebiotics, such as inulin, FOS, and GOS selectively increases the population of Bifidobacterium, a bifidogenic effect similar to that caused by AL and AP in this study [3-5].

There is growing evidence that gut microbial metabolites are key factors influencing host health. Treating rodents with probiotic bacteria of the genera Bifidobacterium and Lactobacillus increased gut $\gamma$-aminobutyric acid (GABA) levels, decreasing depression-like behavior and visceral pain $[17,18]$. Our previous study also showed that AP supplementation markedly increased gut microbial metabolites of amino acids, such as GABA and taurine, which are putative gut-protectors [11]. Bifidobacterium can metabolize linoleic acid into conjugated linoleic acids (CLAs), exerting several putative health-promoting activities, including anticarcinogenic, antiadipogenic, and antidiabetogenic, anti-inflammatory, and antioxidant actions [19]. A recent study has demonstrated that treatment with inulin stimulated Bifidobacterium-mediated the conversion of linoleic acid to CLAs in a human intestinal model [20]. From these findings, we believe that the intake of the Aspergillusderived enzymes, such as AL and AP, is a novel approach to promote the hydrolysis of undigested macronutrients (proteins and lipids) in the intestine to nutrients usable by the gut microbiota, resulting in an elevated abundance of Bifidobacterium and bioactive microbial metabolites. Thus, a metabolomics study is necessary to evaluate the impact of supplemental AL and AP on gut microbial metabolites.

\subsection{Collinsella}

There is accumulating evidence that treatment with inulin and oligosaccharides increases the abundances of Collinsella and Bifidobacterium in the guts of rats [21]. In this study, treatment with AL and AP increased the levels of Collinsella, showing that AL and AP are similar to prebiotic oligosaccharides concerning their effect on Collinsella. Collinsella species appear to be beneficial to health; their increased abundance following dietary supplementation with oligofructose-enriched inulin in obese women is associated with an improved profile of a microbial co-metabolite, hippurate, indicating a healthier phenotype [22]. Collinsella exists in lower concentrations in patients with IBD or chronic pancreatitis than healthy controls [23]. A study by Saalman et al. [24] suggested the potential usefulness of this genus for treating IBD. These studies suggest that Collinsella is beneficial; however, abundance of Collinsella is reportedly positively associated with some disorders, such as type 2 diabetes and rheumatoid arthritis $[25,26]$. Chen et al. showed that Collinsella contributes to pro-inflammatory cytokine production and increased gut permeability in arthritis model mice and Caco-2 cell lines, implying potential adverse effects on some diseases [25]. Altogether, studies regarding the role of Collinsella species in host health are as yet inconclusive.

\subsection{Enterococcus}

This study indicated a higher abundance of Enterococcus in rats fed AL and AP. Enterococcus faecalis exerts positive effects on host health [27] and has clinical relevance for the treatment of chronic recurrent bronchitis [28]. Some members of Enterococcus are used as probiotics and in the production of feed additives to prevent diarrhea or to improve growth in animals [27]. Interestingly, several Enterococcus spp. from food exert antioxidant activity [29]. Studies have indicated that prebiotic oligosaccharides increase the abundance of Enterococcus species in mice and perioperative colorectal cancer patients [30,31]. In this study, both enzyme preparations acted similarly to prebiotic oligosaccharides concerning their effect on Enterococcus. However, Enterococcus species are a leading cause of hospitalassociated bacteremia, endocarditis, and urinary tract infections [32]; they exist in patients with IBD [33]. Despite their pathogenic potential, commensal enterococci display low levels of virulence because they have been safely used for decades as probiotics in humans 
and farm animals [27]. Further research is required to carefully evaluate the implication of the increased abundances of individual members of Enterococcus seen in rats receiving the enzyme preparations used in this study.

\subsection{Oscillospira, Dorea, and Coprobacillus}

It is interesting that treatment with $\mathrm{AL}$ and $\mathrm{AP}$ markedly decreased the relative abundance of Oscillospira, Dorea, and Coprobacillus. Similarly, there is accumulating evidence that consuming various prebiotic oligosaccharides decreases gut abundance of Oscillospira [34,35], Dorea [35,36], and Coprobacillus [37,38] in mice, rats, and dogs. Hitherto, the impact of these genera on host health may depend on the disease type, and further studies are necessary to understand the implication of lower abundance of the genera by $\mathrm{AL}$ and $\mathrm{AP}$.

\subsection{Parabacteroides and Allobaculum}

In this study, the relative abundances of Parabacteroides and Allobaculum were also significantly decreased by AL and AP. So far, studies on the prebiotic oligosaccharides on gut levels of these genera are limited, and controversial. Presently, the implication of lower abundance of Parabacteroides by AL and AP is unknown, and remains to be explored.

\subsection{Bacterial Diversity}

Unexpectedly, in this study, treatment with AL and AP significantly lowered bacterial diversity compared to control. Microbial diversity is considered beneficial for community stability and host health $[39,40]$. However, this may not always be the case, and assumptions of increased diversity could be oversimplified for complicated interactive mechanisms of health and disease [41]. We believe the reduced bacterial diversity in the AL and AP groups might relate to the depletion of many bacterial species, including Parabacteroides, Allobaculum, Oscillospira, Dorea, and Coprobacillus (Figures 2 and 4).

\subsection{Organic Acids}

In this study, it is interesting that AL and AP strikingly increased cecal lactate levels, which were significantly associated with the bifidogenic effects. Meanwhile, the levels of other organic acids, such as acetate, propionate, and butyrate, were decreased by AL and AP. Notably, there was a strong inverse association between lactate and propionate levels. Propionate is microbially produced from lactate in the human colon [42]. Accordingly, the metabolic conversion of lactate into propionate might be lowered in the AL and AP groups. Previous in vitro study indicated free radical scavenging and antioxidant effects of lactate [43]. Recent studies have suggested that lactate exhibits an inflammatory or anti-inflammatory role depending on its effects on immune cell types and disease types [44]. Thus, the implication of lactate accumulation in the AL and AP groups remains to be explored.

\subsection{Limitations of this Study}

Study limitations should be highlighted. In this study, both AL and AP used were crude enzyme preparations and contained minor components other than lipase and protease. Thus, other factors, except for lipase and protease in these preparations, might have contributed to the effects observed in the gut microbiota. Further research is recommended to investigate the impact of highly purified lipase and protease obtained from Aspergillus species on intestinal microbiota. In addition, this study used wild-type rats, but not diseasemodel rats. Thus, the effects of Aspergillus lipase and protease on disease-model animals remains to be elucidated.

\section{Conclusions}

This study is the first to show the powerful bifidogenic effect of $\mathrm{AL}$, an impact that, interestingly, was far stronger than that of AP. Additionally, this study specifically revealed 
the modulations of the relative abundances of other species of the genera Collinsella, Enterococcus, Oscillospira, Dorea, and Coprobacillus by the enzyme preparations. These modulations were similar to those reported in several studies with typical prebiotic oligosaccharides. The results might provide novel insights into the impacts of Aspergillus protease and lipase, and of Aspergillus-fermented foods containing Aspergillus proteases and lipases on gut health. Additionally, our studies might introduce a new concept of "prebiotic digestive enzymes" based on the findings of the impacts of dietary Aspergillus lipase and protease on gut Bifidobacterium. Our findings raise a fundamental question of how exogenous digestive enzymes of fats and proteins lead to their bifidogenic effects. Further studies will address this question. Metabolomics study in our group is in progress to investigate the gut metabolites derived from dietary fats and proteins in rats fed AL and AP to understand the impacts of AL and AP on the gut health.

Supplementary Materials: The following are available online at https:/ / www.mdpi.com/article/ 10.3390 / fermentation7040294/s1. Table S1. Composition of basal diet. Figure S1. Correlation matrix (correlation coefficient, $r$ ) between cecal levels of organic acids and microbiota composition. The deep color bars indicate correlation strength. ${ }^{*} p<0.05,{ }^{* *} p<0.01$.

Author Contributions: Conceptualization, Investigation, Methodology, Validation, Writing—original draft: Y.Y., T.K. and N.K.; Formal analysis, Software, Methodology, Visualization: Y.Y., T.K., S.F., M.K. and S.Y.; literature search: Y.Y., N.K.; Project administration, Funding acquisition, Supervision, Writing-review and editing: N.K., T.K., S.F., M.K. and S.Y. All authors have read and agreed to the published version of the manuscript.

Funding: This study was supported in part by the grant from the HIRAKU consortium, Hiroshima University (to T.K.). Amano Enzyme Inc. (to N.K.), JSPS KAKENHI (18H04805 to S.F.), AMED-CREST (JP20gm1010009 to S.F.), JST ERATO (JPMJER1902 to S.F.), the Takeda Science Foundation (to S.F.), the Food Science Institute Foundation (to S.F.), and the Program for the Advancement of Research in Core Projects under Keio University's Longevity Initiative (to S.F.).

Institutional Review Board and Statement: The study was conducted according to the guidelines of the Declaration of Helsinki, and approved by the Ethics Committee of the Hiroshima University (Ethical approval No. C15-12), approved on 16 June 2015.

Data Availability Statement: The data of the 16S rRNA gene sequences of gut microbiota presented in this study can be available from the DDBJ database (http:/ / getentry.ddbj.nig.ac.jp/) (accessed 21 January 2021) under accession number DRA011374. Further inquiries can be directed to the corresponding authors.

Conflicts of Interest: This study received funding from Amano Enzyme Inc. The funders had no role in the design of the study, in the collection, analyses, or interpretation of data, in the writing of the manuscript, or in the decision to publish the results. All authors declare no other competing interests in the current study.

\section{References}

1. Hasan, N.; Yang, H. Factors affecting the composition of the gut microbiota, and its modulation. PeerJ 2019, 7, e7502. [CrossRef] [PubMed]

2. Conlon, M.A.; Bird, A.R. The impact of diet and lifestyle on gut microbiota and human health. Nutrients 2015, 7, 17-44. [CrossRef]

3. Patel, S.; Goyal, A. The current trends and future perspectives of prebiotics research: A review. 3 Biotech 2012, 2, 115-125. [CrossRef]

4. Tsai, Y.L.; Lin, T.L.; Chang, C.J.; Wu, T.R.; Lai, W.F.; Lu, C.C. Probiotics, prebiotics and amelioration of diseases. J. Biomed. Sci. 2019, 26, 3. [CrossRef]

5. Stavropoulou, E.; Bezirtzoglou, E. Probiotics in Medicine: A Long Debate. Front. Immunol. 2020, 11, 2192. [CrossRef] [PubMed]

6. Ichishima, E. Enzymology of the national microorganisms of Japan in a historical context. J. Biochem. Biotechnol. 2019, 2, 11-18.

7. Takenaka, S.; Nakabayashi, R.; Ogawa, C.; Kimura, Y.; Yokota, S.; Doi, M. Characterization of surface Aspergillus community involved in traditional fermentation and ripening of katsuobushi. Int. J. Food Microbiol. 2020, 327, 108654. [CrossRef]

8. Ianiro, G.; Pecere, S.; Giorgio, V.; Gasbarrini, A.; Cammarota, G. Digestive Enzyme Supplementation in Gastrointestinal Diseases. Curr. Drug Metabol. 2016, 17, 187-193. [CrossRef] 
9. Yang, Y.; Sitanggang, N.V.; Kato, N.; Inoue, J.; Murakami, T.; Watanabe, T.; Iguchi, T.; Okazaki, Y. Beneficial effects of protease preparations derived from Aspergillus on the colonic luminal environment in rats consuming a high-fat diet. Biomed. Rep. 2015, 3, 715-720. [CrossRef]

10. Yang, Y.; Iwamoto, A.; Kumrungsee, T.; Okazaki, Y.; Kuroda, M.; Yamaguchi, S.; Katoa, N. Consumption of an acid protease derived from Aspergillus oryzae causes bifidogenic effect in rats. Nutr. Res. 2017, 44, 60-66. [CrossRef]

11. Yang, Y.; Kumrungsee, T.; Kuroda, M.; Yamaguchi, S.; Kato, N. Feeding Aspergillus protease preparation combined with adequate protein diet to rats increases levels of cecum gut-protective amino acids, partially linked to Bifidobacterium and Lactobacillus. Biosci. Biotech. Biochem. 2019, 83, 1901-1911. [CrossRef] [PubMed]

12. Van Hul, M.; Karnik, K.; Canene-Adams, K.; De Souza, M.; Abbeele, P.V.D.; Marzorati, M.; Delzenne, N.M.; Everard, A.; Cani, P.D. Comparison of the effects of soluble corn fiber and fructooligosaccharides on metabolism, inflammation, and gut microbiome of high-fat diet-fed mice. Am. J. Physiol. Endocrinol. Metab. 2020, 319, E779-E791. [CrossRef]

13. Ishii, C.; Nakanishi, Y.; Murakami, S.; Nozu, R.; Ueno, M.; Hioki, K.; Aw, W.; Hirayama, A.; Soga, T.; Ito, M.; et al. A metabologenomic approach reveals changes in the intestinal environment of mice fed on American diet. Int. J. Mol. Sci. 2018, 19, 4079. [CrossRef] [PubMed]

14. Magoč, T.; Salzberg, S.L. FLASH: Fast length adjustment of short reads to improve genome assemblies. Bioinformatics 2011, 27, 2957-2963. [CrossRef]

15. Zhang, S.; Wang, H.; Zhu, M. A sensitive GC/MS detection method for analyzing microbial metabolites short chain fatty acids in fecal and serum samples. Talanta 2019, 196, 249-254. [CrossRef] [PubMed]

16. Hooijmans, C.R.; Rovers, M.M.; BM de Vries, R.; Leenaars, M.; Ritskes-Hoitinga, M.; Langendam, M.W. SYRCLE's risk of bias tool for animal studies. BMC Med. Res. Meth. 2014, 14, 43. [CrossRef]

17. Patterson, E.; Ryan, P.M.; Wiley, N.; Carafa, I.; Sherwin, E.; Moloney, G.; Franciosi, E.; Mandal, R.; Wishart, D.S.; Tuohy, K.; et al. Gamma-aminobutyric acid-producing lactobacilli positively affect metabolism and depressive-like behaviour in a mouse model of metabolic syndrome. Sci. Rep. 2019, 9, 16323. [CrossRef]

18. Pokusaeva, K.; Johnson, C.; Luk, B.; Uribe, G.; Fu, Y.; Oezguen, N.; Matsunami, R.K.; Lugo, M.; Major, A.; Mori-Akiyama, Y.; et al. GABA-producing Bifidobacterium dentium modulates visceral sensitivity in the intestine. Neurogastroenterol. Motil. 2017, 29, e12904. [CrossRef]

19. Basak, S.; Duttaroy, A.K. Conjugated Linoleic Acid and Its Beneficial Effects in Obesity, Cardiovascular Disease, and Cancer. Nutrients 2020, 12, 1913. [CrossRef]

20. Carafa, I.; Masuero, D.; Vrhovsek, U.; Bittante, G.; Franciosi, E.; Tuohy, K.M. Production of conjugated linoleic acid (CLA): Effect of inulin on microbial composition and CLA concentration in a human intestinal model. Proc. Nutr. Soc. 2020, 79 (OCE2), E628. [CrossRef]

21. Klancic, T.; Laforest-Lapointe, I.; Choo, A.; Nettleton, J.E.; Chleilat, F.; Noye Tuplin, E.W.; Alukic, E.; Cho, N.A.; Nicolucci, A.C.; Arrieta, M.; et al. Prebiotic Oligofructose Prevents Antibiotic-Induced Obesity Risk and Improves Metabolic and Gut Microbiota Profiles in Rat Dams and Offspring. Mol. Nutr. Food Res. 2020, 64, e2000288. [CrossRef]

22. Dewulf, E.M.; Cani, P.D.; Claus, S.P.; Fuentes, S.; Puylaert, P.G.B.; Neyrinck, A.M.; Bindels, L.B.; de Vos, W.M.; Gibson, G.R.; Thissen, J.; et al. Insight into the prebiotic concept: Lessons from an exploratory, double blind intervention study with inulin-type fructans in obese women. Gut 2013, 62, 1112-1121. [CrossRef] [PubMed]

23. Kassinen, A.; Krogius-Kurikka, L.; Mäkivuokko, H.; Rinttilä, T.; Paulin, L.; Corander, J.; Malinen, E.; Apajalahti, J.; Palva, A. The Fecal Microbiota of Irritable Bowel Syndrome Patients Differs Significantly From That of Healthy Subjects. Gastroenterology 2007, 133, 24-33. [CrossRef] [PubMed]

24. Saalman, R.; Alderberth, I.; Wold, A.; Sjoberg, F. Use of Collinsella for Treatment of Inflammatory Bowel Disease. U.S. Patent No. 2018/0028579 A1, 1 February 2018.

25. Chen, J.; Wright, K.; Davis, J.M.; Jeraldo, P.; Marietta, E.V.; Murray, J.; Nelson, H.; Matteson, E.L.; Taneja, V. An expansion of rare lineage intestinal microbes characterizes rheumatoid arthritis. Genome Med. 2016, 8, 43. [CrossRef] [PubMed]

26. Shah, V.; Lambeth, S.M.; Carson, T.; Lowe, J.; Ramaraj, T.; Leff, J.W.; Luo, L.; Bell, J.C.; Shah, V.O. Composition Diversity and Abundance of Gut Microbiome in Prediabetes and Type 2 Diabetes. J. Diabetes Obes. 2015, 2, 1-7. [CrossRef]

27. Hanchi, H.; Mottawea, W.; Sebei, K.; Hammami, R. The Genus Enterococcus: Between Probiotic Potential and Safety Concerns-An Update. Front. Microbiol. 2018, 9, 1791. [CrossRef]

28. Habermann, W.; Zimmermann, K.; Skarabis, H.; Kunze, R.; Rusch, V. The effect of a bacterial immunostimulant (human Enterococcus faecalis bacteria) on the occurrence of relapse in patients with chronic bronchtis. Arzneimittelforschung 2001, 51, 931-937.

29. Pieniz, S.; Andreazza, R.; Anghinoni, T.; Camargo, F.; Brandelli, A. Probiotic potential, antimicrobial and antioxidant activities of Enterococcus durans strain LAB18s. Food Control 2014, 37, 251-256. [CrossRef]

30. Mao, B.Y.; Li, D.Y.; Zhao, J.X.; Liu, X.M.; Gu, Z.N.; Chen, Y.Q.; Zhang, H.; Chen, W. Metagenomic insights into the effects of fructo-oligosaccharides (FOS) on the composition of fecal microbiota in mice. J. Agric. Food Chem. 2015, 63, 856-863. [CrossRef] [PubMed]

31. Xie, X.; He, Y.; Li, H.; Yu, D.; Na, L.; Sun, T.; Zhang, D.; Shi, X.; Xia, Y.; Jiang, T.; et al. Effects of prebiotics on immunologic indicators and intestinal microbiota structure in perioperative colorectal cancer patients. Nutrition 2019, 61, 132-142. [CrossRef]

32. Dubin, K.; Pamer, E.G. Enterococci and Their Interactions with the Intestinal Microbiome. Microbiol. Spectrum 2014, 5, 5-6. 
33. Lo Presti, A.; Zorzi, F.; Del Chierico, F.; Altomare, A.; Cocca, S.; Avola, A.; de Biasio, F.; Russo, A.; Cella, E.; Reddel, S.; et al. Fecal and Mucosal Microbiota Profiling in Irritable Bowel Syndrome and Inflammatory Bowel Disease. Front. Microbiol. 2019, 10, 1655. [CrossRef]

34. Fei, Y.; Wang, Y.; Pang, Y.; Wang, W.; Zhu, D.; Xie, M.; Lan, S.; Wang, Z. Xylooligosaccharide Modulates Gut Microbiota and Alleviates Colonic Inflammation Caused by High Fat Diet Induced Obesity. Front. Physiol. 2020, 10, 1601. [CrossRef] [PubMed]

35. Wang, L.; Pan, M.; Li, D.; Yin, Y.; Jiang, T.; Fang, S.; Wang, G.; Zhao, J.; Zhang, H.; Chen, W. Metagenomic insights into the effects of oligosaccharides on the microbial composition of cecal contents in constipated mice. J. Funct. Foods 2017, 38, 486-496. [CrossRef]

36. Garcia-Mazcorro, J.F.; Barcenas-Walls, J.R.; Suchodolski, J.S.; Steiner, J.M. Molecular assessment of the fecal microbiota in healthy cats and dogs before and during supplementation with fructo-oligosaccharides (FOS) and inulin using high-throughput 454-pyrosequencing. Peer] 2017, 5, e3184. [CrossRef] [PubMed]

37. Alexander, C.; Cross, T.-W.L.; Devendran, S.; Neumer, F.; Theis, S.; Ridlon, J.M.; Suchodolski, J.S.; de Godoy, M.R.C.; Swanson, K.S. Effects of prebiotic inulin-type fructans on blood metabolite and hormone concentrations and faecal microbiota and metabolites in overweight dogs. Br. J. Nutr. 2018, 120, 711-720. [CrossRef] [PubMed]

38. Everard, A.; Lazarevic, V.; Gaïa, N.; Johansson, M.; Ståhlman, M.; Bäckhed, F.; Delzenne, N.; Schrenzel, J.; Francois, P.; Cani, P. Microbiome of prebiotic-treated mice reveals novel targets involved in host response during obesity. ISME J. 2014, 8, 2116-2130. [CrossRef] [PubMed]

39. Audebert, C.; Even, G.; Cian, A.; Loywick, A.; Merlin, S.; Viscogliosi, E.; Chabe, M.; Blastocystis Investigation Group. Colonization with the Enteric Protozoa Blastocystis is Associated with Increased Diversity of Human Gut Bacterial Microbiota. Sci. Rep. 2016, 6, 25255. [CrossRef]

40. Sjöberg, F.; Barkman, C.; Nookaew, I.; Östman, S.; Adlerberth, I.; Saalman, R.; Wold, A.E. Low-complexity microbiota in the duodenum of children with newly diagnosed ulcerative colitis. PLoS ONE 2017, 12, e0186178. [CrossRef]

41. Shade, A. Diversity is the question, not the answer. ISME J. 2017, 11, 1-6. [CrossRef]

42. Flint, H.J.; Duncan, S.H.; Scott, K.P.; Louis, P. Links between diet, gut microbiota composition and gut metabolism. Proc. Nutr. Soc. 2015, 74, 13-22. [CrossRef] [PubMed]

43. Groussard, C.; Morel, I.; Chevanne, M.; Monnier, M.; Cillard, J.; Delamarche, A. Free radical scavenging and antioxidant effects of lactate ion: An in vitro study. J. Appl. Physiol. 2000, 89, 169-175. [CrossRef] [PubMed]

44. Manoharan, I.; Prasad, P.D.; Thangaraju, M.; Manicassamy, S. Lactate-Dependent Regulation of Immune Responses by Dendritic Cells and Macrophages. Front. Immunol. 2021, 12, 691134. [CrossRef] [PubMed] 